\title{
El conocimiento como recurso estratégico para el aprendizaje interorganizativo
}

\author{
Benavides Espinosa, María del Mar*
}

\section{Resumen}

Las empresas se enfrentan a un entorno muy competitivo, en donde la posesión de conocimiento es vital para su supervivencia. Estamos en la era del conocimiento. En este sentido, el presente artículo plantea dos objetivos, primero plantea analizar las características del conocimiento relevantes para considerarlo como recurso estratégico, como son la complejidad, la codificación y la especificidad. Y en el segundo objetivo, se verifica la influencia de dichas características en el aprendizaje de conocimientos entre empresas a través de una cooperación, como puede ser una joint venture. Para ello, por un lado, se estudiaron sus dimensiones, usando una epistemología pluralista, y por otro, las características del recurso estratégico en el marco de la Teoría de Recursos y Capacidades, estableciendo relaciones entre ellas. Para contrastar las hipótesis planteadas, se utilizaron las ecuaciones estructurales, obteniendo como resultado que uno de los aspectos más críticos del conocimiento estratégico es su complejidad, y más, si está poco codificado. Concluimos que la joint venture es un instrumento que facilita el aprendizaje, superando así el obstáculo de la complejidad.

Palabras clave: Joint venture, Aprendizaje, Conocimiento, Teoría de Recursos y Capacidades, Ecuaciones Estructurales.

\section{Knowledge as a Strategic Resource for Interorganizational Learning}

\begin{abstract}
Companies face a very competitive environment, in which possession of knowledge is vital for their survival; we live in the age of knowledge. This article has two objectives: the first is to analyze the characteristics of knowledge, such as complexity, codification and specificity, relevant to considering it as a strategic resource. The second objective is to verify the influence of the above-mentioned characteristics on learning between companies through cooperation, as in a joint venture. On the one hand, the dimensions of the characteristics were studied using a pluralist epistemology, and on the
\end{abstract}

Recibido: 01-02-11 Aceptado: 01-09-11

* Doctora en Economía. Docente del Departamente de Dirección de Empresas de la Universitat de Valência. e-mail: maria.m.benavides@uv.es 
other, characteristics of the strategic resource were examined using the framework of the Theory of Resources and Capabilities, establishing relations among them. To contrast the proposed hypotheses, structural equations were used obtaining the result that one of the most critical aspects of strategic knowledge is its complexity, even more so if it is not highly codified. Conclusions are that the joint venture is an instrument that facilitates learning, overcoming the obstacle of complexity.

Key words: Joint venture, learning, knowledge, theory of resources and capabilities, structural equations.

\section{Introducción}

En el actual contexto económico, el conocimiento se convierte en un activo clave. En este sentido, la literatura ha puesto de manifiesto la importancia del conocimiento, especialmente, para aquellas empresas que necesitan innovar con el fin de mantener y mejorar la ventaja competitiva (Lin, 2007; Huang et al., 2008). La concepción de la empresa basada en el conocimiento (Nonaka, 1994; Spender, 1994; Grant, 1996) es la consideración de la empresa como un almacén de conocimientos, capaz de generar y aplicar nuevo conocimiento; esta concepción es una perspectiva interesante para la competitividad de la empresa.

En este trabajo se plantean dos objetivos. El primero de ellos es analizar las dimensiones y las características que tiene un conocimiento para ser considerado estratégico, y por lo tanto, ser capaz de generar una ventaja competitiva. Por un lado, se analiza las dimensiones de la complejidad, codificación y especificidad del conocimiento (Badaracco, 1992), y por otro, según la Teoría de Recursos y Capacidades (Peteraf, 1993; Amit y Schoemaker, 1993) las características según el grado de movilidad, asimilación, e imitación, tal que si es difícil de transferir e imitar hará que la empresa que lo posea pueda mantener esa ventaja competitiva, en la medida que ese conocimiento no sea adquirido por otras empresas.

Por otra parte, en el segundo objetivo, se verifica la influencia de las características del conocimiento en el aprendizaje de los mismos mediante una cooperación entre empresas, como lo es una joint venture. Existen varias alternativas para adquirir conocimiento estratégico, la cooperación, la fusión y la adquisición. En este artículo, se plantea como una opción eficiente, la cooperación, pues es una opción rápida y temporal, que permite a la empresa que aprende una mayor flexibilidad que el resto de las opciones. Además, dentro de los distintos tipos de cooperación, hay unas formas más adecuadas que otras para el aprendizaje de dichos conocimientos estratégicos. En este trabajo, se expone que la joint venture es una de las pocas opciones válidas para que una empresa pueda adquirir este tipo de conocimiento (Pak y Park, 2004; Benavides, 2007; Lyles y Salk, 2007; Wahab et al., 2010), y luego, los pueda utilizar para otros uso distintos.

Para poder llevar a cabo esta investigación fue necesario seleccionar una muestra de empresas que hubieran realizado al menos una joint venture. Del total de empresas de la base de datos, finalmente, la muestra obtenida es de 74 . Son muy escasos los trabajos de tipo cuantitativos debido a la dificultad de conseguir una muestra de estas característi- 
El conocimiento como recurso estratégico para el aprendizaje interorganizativo Benavides Espinosa, María del Mar

cas. En éste, abordamos un vacío de investigación existente tanto en la generación de escalas fiables y válidas sobre el aprendizaje y conocimiento a través de la joint venture, para poder contrastar las relaciones propuestas de las hipótesis, como el análisis de estas relaciones con un modelo estructural.

\section{El conocimiento en las organizaciones como un recurso estratégico}

Desde un punto de vista epistemológico, el conocimiento hace énfasis en lo absoluto y lo estático, expresado en proposiciones y fórmulas lógicas. Ahora bien, una definición clásica de conocimiento es la creencia verdadera justificada. La creencia y la verdad son condiciones necesarias para el saber (Blasco y Grimaltos, 1997).

Dentro de un contexto organizativo, Cohen y Levinthal (1990) definen el conocimiento como un conjunto de habilidades de aprendizaje, como la acumulación de "saberes", entendimientos o razonamientos que se van formando dentro de sus límites, dependiendo de la capacidad de que disponen las organizaciones. El conocimiento puede, por lo tanto, ser distinguido desde la opinión, especulación y creencias, u otros tipos de información no probadas. De forma más concreta, Kogut y Zander (1992) señalan que el conocimiento es el resultado de dos componentes: la información y el know-how (habilidad, saber cómo).

Existen distintos tipos de conocimiento, según la variable que se analice. La clasificación más utilizada en la literatura del conocimiento es la distinción en- tre conocimiento tácito o implícito y el conocimiento explícito. Inkpen y Dinur (1998) parten de que el conocimiento es un continuo donde los extremos son, por un lado, el conocimiento explícito y, por el otro, el conocimiento implícito. Tal que, el conocimiento explícito está codificado, es decir, tiene poco o nada de componente tácito, y el conocimiento implícito, no está codificado. Entre estos dos extremos se encuentran "conocimientos híbridos" compuestos por una parte explícita y por otra parte tácita, que marcan una amplia gama de conocimientos, en función de los distintos grados de sus componentes. Como base hacia una teoría de conocimiento se utiliza una epistemología múltiple o pluralista, y para ello, se clasificaron los distintos tipos de conocimientos, que se resume en el Cuadro 1.

A diferencia de los recursos tangibles, el conocimiento puede estar en varios sitios a la vez, en fórmulas, en diseños, en manuales, en libros o piezas de maquinaria, incluso, en los mismos productos. Por ejemplo, dependiendo del grado de componente tácito que posea, se podrá transmitir con más o menos facilidad. La rapidez con la que el conocimiento emigra no depende sólo de sus características intrínsecas, sino también, de otras variables que pueden acelerar o frenar su transferencia. Según la Teoría de Recursos y Capacidades, para analizar el conocimiento como recurso estratégico, se debe estudiar la heterogeneidad, la movilidad y su dificultad de imitación, como las características que así lo determinan.

Una organización es heterogénea en cuanto a su dotación de conocimientos y a los usos específicos que se hacen 


\section{Cuadro 1}

Tipos de conocimientos.

\begin{tabular}{|c|c|c|c|}
\hline \multirow{2}{*}{\begin{tabular}{l}
\multicolumn{1}{c}{ En función de } \\
Grado de \\
especificidad
\end{tabular}} & \multicolumn{2}{|c|}{ Tipos de Conocimientos } & \multirow{2}{*}{\begin{tabular}{l}
\multicolumn{1}{c}{ Autores } \\
Jensen y Meckling, \\
1992; Wruch y Jense, \\
1994
\end{tabular}} \\
\hline & $\begin{array}{l}\text { Genérico que puede } \\
\text { aplicarse a muchas } \\
\text { organizaciones }\end{array}$ & $\begin{array}{l}\text { Específico tiene valor } \\
\text { solo para algunas } \\
\text { organizaciones }\end{array}$ & \\
\hline \multirow[t]{2}{*}{ Grado de codificación } & $\begin{array}{l}\text { Explícito que es } \\
\text { transmisible, de gran } \\
\text { movilidad, fácilmente } \\
\text { procesado por un } \\
\text { ordenador y } \\
\text { almacenado }\end{array}$ & $\begin{array}{l}\text { Tácito, implícito, } \\
\text { no verbalizable; } \\
\text { Difícil de comunicar, } \\
\text { se adquiere viendo } \\
\text { hacer o haciendo. } \\
\text { No articulable }\end{array}$ & $\begin{array}{l}\text { Polanyi, 1967; Itami y } \\
\text { Roehl 1987; Hedlund, } \\
\text { 1994; Winter, 1987; } \\
\text { Nonaka y Takeuchi, } \\
\text { 1995; Kogut y Zander, } \\
\text { 1992; Inkpen, 1998. } \\
\text { Subramaniam y } \\
\text { Venkatraman (2001); } \\
\text { Pak y Park, (2004); } \\
\text { Minbaeva (2007) }\end{array}$ \\
\hline & $\begin{array}{l}\text { Migratorio, que se } \\
\text { mueve rápida y } \\
\text { fácilmente }\end{array}$ & $\begin{array}{l}\text { Insertado que se } \\
\text { mueve con lentitud, y } \\
\text { reside en las } \\
\text { relaciones sociales }\end{array}$ & Badaraco, 1992 \\
\hline \multirow[t]{2}{*}{ Grado de complejidad } & $\begin{array}{l}\text { Observable, no } \\
\text { complejo que puede } \\
\text { ser revelado con el } \\
\text { uso o utilización }\end{array}$ & $\begin{array}{l}\text { No observable, } \\
\text { complejo, que el } \\
\text { grado de revelación } \\
\text { no se produce con su } \\
\text { utilización }\end{array}$ & $\begin{array}{l}\text { Winter, } 1987 \\
\text { Subramaniam y } \\
\text { Venkatraman (2001) }\end{array}$ \\
\hline & $\begin{array}{l}\text { Comprendido } \\
\text { simpatizado, explicito } \\
\text { socializado } \\
\text { Operacional, } \\
\text { basado en la } \\
\text { experiencia, vía } \\
\text { internalización }\end{array}$ & $\begin{array}{l}\text { Conceptual, } \\
\text { resultante de la } \\
\text { externalización de } \\
\text { conocimientos } \\
\text { Sistémico } \\
\text { Requiere de otros } \\
\text { conocimientos } \\
\text { relacionados }\end{array}$ & $\begin{array}{l}\text { Winter, 1987; Nonaka } \\
\text { y Takeuchi, } 1995\end{array}$ \\
\hline
\end{tabular}

Fuente: Elaboración propia.

de ellos. Cuanto más específico es su uso, más difícil será su utilización para otras aplicaciones. Por ello, el conocimiento tiene un valor económico que vendrá dado en función de su utilidad, y también, de su rareza, por lo que cuanto más escasos y útiles, mayor heterogeneidad.
El conocimiento puede no estar codificado, pero puede ser observado. La codificación, por una parte, facilita la transferencia interna en la organización, pero, por otra parte, tiene el inconveniente de ser más accesible para la competencia. Así pues, cuanto menos codificable sea, menos movible o transferible. 
El conocimiento como recurso estratégico para el aprendizaje interorganizativo Benavides Espinosa, María del Mar

Por último, la difícil imitación de los recursos, dependerá de los mecanismos aislantes (Rumelt, 1984, 1987) que utilizan las organizaciones para protegerse de la competencia y mantener sus rentas durante el mayor tiempo posible. Algunos de estos mecanismos son: la posesión de marcas y patentes, o derechos de propiedad, existencia de barreras de entrada y del resultado de la experiencia en competir. También, la complejidad técnica del conocimiento constituye una barrera a la imitación (Dierickx y Cool, 1989; Reed y DeFillippi, 1990; Simonin, 1999) dada su relación con otros conocimientos. Así como, la complejidad social, que al ser el conocimiento dependiente de las interrelaciones entre los trabajadores, y por lo tanto, dependiente de las relaciones del sistema, es más difícil de trasladarlo a otras empresas.

Se observa que existen ciertas relaciones entre las dimensiones del conocimiento y las características del conocimiento como recurso estratégico. Respecto a la especificidad, en sí, no es una dimensión propiamente dicha, sino que determina qué conocimiento es, con su contenido. Esta idea está asociada a la heterogeneidad de los recursos para ser estratégicos, pues normalmente cuanto más específico es, mayor variedad de recursos habrá en una empresa debido a la limitación de su uso. La codificación entendida como la habilidad para estructurar el conocimiento, está relacionada con la facilidad para su transferencia, y por lo tanto, de su movilidad. Y la complejidad, está directamente relacionada con la imitación, especialmente en su aspecto más técnico, pues la complejidad social está relacionada con la imitación pero también con la movilidad.

Respecto al aprendizaje de conocimientos entre empresas, éste se ve afectado por el grado de codificación y complejidad, como verdaderas dimensiones del conocimiento, sin embargo, el grado de especificidad de un conocimiento concreto no influye en la transferencia de forma significativa. Por otra parte, la codificación y la complejidad son dimensiones que están relacionadas entre si, aunque no son barreras permanentes; pues, cuanto más complejo es un conocimiento, más difícil es de codificar y, por lo tanto, de transmitir. Así pues, los factores clave para la transferencia de conocimientos son la codificación y la complejidad.

Por un lado, el conocimiento explícito, al estar codificado facilita su transferencia, sea poco o muy complejo. Evidentemente, el conocimiento poco complejo será más fácil de transferir, mientras que un conocimiento muy complejo será más difícil de transferir, pero no imposible. Sin embargo, un conocimiento tácito, o no codificado, podrá ser adquirido con relativa facilidad, si puede ser observado, pero si además, es complejo, necesita que éste sea comprendido y asimilado. A continuación, se resumen estas relaciones en el Cuadro 2.

Disponer de un conocimiento, no garantiza de por sí su utilización, el emisor debe saber utilizar el conocimiento que ha adquirido. Los conocimientos estratégicos principalmente suelen ser conocimientos complejos, y no suelen estar demasiado codificados, por lo que son difíciles de ser transferidos sin ayuda de quien los posee. Que un conocimiento 
Cuadro 2

Relación entre la complejidad y la codificación del conocimiento.

\begin{tabular}{ccc}
\hline \multicolumn{1}{c}{ Conocimiento } & Codificado & Tácito \\
\hline Poco complejo & $\begin{array}{c}\text { Muy fácil de transferir } \\
\text { Muy codificado y poco complejo }\end{array}$ & $\begin{array}{c}\text { Fácil de transferir } \\
\text { Poco codificado y poco } \\
\text { complejo }\end{array}$ \\
Muy complejo & Difícil de transferir & Muy difícil de transferir \\
& Muy codificado y muy complejo Poco codificado y muy complejo \\
\hline
\end{tabular}

Fuente: Elaboración propia.

esté más o menos codificado, que sea más o menos tácito, es una dimensión que facilita u obstaculiza la transferencia pero no la impide, no así la complejidad.

\section{Las dimensiones del conocimiento para su aprendizaje mediante la joint venture}

En este apartado, se analiza la influencia de las distintas dimensiones del conocimiento en el aprendizaje realizado entre los socios de una cooperación, en este caso, entre los socios de la joint venture. La adquisición del conocimiento de una empresa respecto de otra, depende de la naturaleza del conocimiento aportado en relación con el conocimiento que posee el socio (Inkpen, 2000; 2008); previamente será necesario que el socio que va a adquirir el nuevo conocimiento tenga un adecuado conocimiento base que le permita la utilización efectiva del conocimiento aprendido del socio (Cohen y Levinthal, 1990; Simonin, 1999). Y además, para adquirir conocimiento de otra organización, éste debe ser como mínimo accesible, cuestión que con la cooperación es posible (Abarca, 2010; Burns, et al., 2011).

\subsection{La codificación del conocimiento transferido en la joint venture}

Se entiende por codificación la habilidad de la organización para estructurar el conocimiento dentro de un tipo de reglas identificadas y de relaciones que pueden ser fácilmente entendibles (Reed y DeFillippi, 1990; Kogut y Zander, 1992), tal que lo hacen fácil para su transferencia (Swift y Hwang, 2008), no así para cuando no lo está (conocimiento tácito).

Si el conocimiento es tácito, no es fácil su transferencia pero éste puede ser entendido mejor o copiado de forma más correcta, si la transferencia de conocimientos se realiza a través de una cooperación o una joint venture (Hennart, 1988), pues implica la colaboración del socio para ser interpretado. La joint venture es una herramienta para aprender conocimientos tácitos de otras empresas, pues hace que este conocimiento sea más accesible para el socio, con la ventaja de que sigue siendo inaccesible para el resto de los competidores.

Por esto, Simonin (1999) dice que el componente tácito es una fuente de inestabilidad o de conflicto en la cooperación, y es una manifestación de la dificul- 
El conocimiento como recurso estratégico para el aprendizaje interorganizativo Benavides Espinosa, María del Mar

tad y frustración en el aprendizaje de conocimientos. Pero en la joint venture, el socio que posee el conocimiento objeto de cooperación tiene el compromiso de facilitar el acceso y su entendimiento a su socio, por lo que minimiza esta inestabilidad, facilitando su comprensión y asimilación, pues puede darse un contacto continuo con el personal experimentado para observar, imitar y practicar los conocimientos tácitos como requiere su aprendizaje. El riesgo del conocimiento tácito es que el conocimiento transferido a la joint venture se disipe. Por lo que se propone la siguiente hipótesis:

Hipótesis 1: El establecimiento de una joint venture facilita la transferencia de conocimiento tácito generando así un mayor aprendizaje de conocimientos estratégicos.

\subsection{La complejidad del conocimiento transferido en la joint venture}

Se entiende por complejidad el número de parámetros que define un sistema, dentro de un orden dado. La complejidad proviene de un gran número de tecnologías, rutinas, y de la experiencia de los individuos o equipos (Reed y DeFillippi, 1990). En general, existen dos conceptos de complejidad claramente diferenciados, la complejidad técnica y la complejidad social. La primera es definida por Simonin (1999) como el número de tecnologías interdependientes, rutinas, individuos y recursos relacionados con un conocimiento. $Y$ la segunda, es el grado en que un determinado conocimiento depende de otros conocimientos (Winter, 1987; Zander y Kogut, 1995; Wonglimpiyarat, 2005; Bianchi et al., 2011), se refiere a las relaciones especializadas entre individuos.

Estudios empíricos como el realizado por Simonin (1999) destaca la gran importancia del componente de la complejidad junto al componente tácito en la transferencia de cocimientos, en contraste con la poca influencia de la especificidad. Más concretamente, Mowery et al. (1996) confirman que la joint venture parece ser más adecuada para la transferencia de conocimientos complejos que los acuerdos de cooperación sin creación de una entidad propia para ello.

La complejidad afecta básicamente a la comprensión del conocimiento que se transmite, y parece estar claro que la joint venture facilita la adquisición y comprensión de conocimiento complejo de otros socios (Inkpen y Beamish, 1997). También, reduce la ambigüedad causal de la transferencia, ya que existe un mayor contacto entre ellos, facilitando así la asimilación de dichos conocimientos. Además, el aprendizaje de los conocimientos complejos será más fácil cuando éste sea el objetivo de la joint venture, pues el grado de compromiso de los socios en conseguir el aprendizaje es elevado para este tipo de cooperación. Así pues, se propone la hipótesis siguiente: Hipótesis 2: El establecimiento de una joint venture facilita la transferencia de conocimiento complejo generando así un mayor aprendizaje de conocimientos estratégicos. 


\section{El conocimiento como recurso estratégico para el aprendizaje interorganizativo a través de una joint venture: Modelo estructural}

Previamente a comprobar el modelo estructural derivado de las relaciones establecidas en las hipótesis propuestas, se determina si las escalas cumplen las propiedades psicométricas (fiabilidad y validez) dentro del modelo propuesto.

Para ello, se realizo un análisis factorial confirmatorio (AFC), en donde se demuestra que los índices de fiabilidad compuesta de las tres escalas alcanzan los niveles satisfactorios al ser mayores de 0.7.

Asimismo, la validez de contenido se comprobó revisando numerosos trabajos teóricos y empíricos, sobre todo, estos últimos para intentar conocer cuáles eran las dimensiones de cada escala. La validez convergente, se determino revisando los estadísticos $t$ de las cargas factoriales para ver cuáles eran significativos. En la Tabla 1, se muestra los resultados que indican, por un lado, unas estimaciones muy buenas con un nivel de significatividad alta (pues todos los estadísticos $t$ son mayores a 3.291 y por consiguiente, significativos para p.001) y ë estandarizados con valores altos, todos ellos muy por encima del 0.4 (Hair et al., 1999).

Y por otro lado, la mayoría de los estadísticos de la bondad del ajuste toman valores más o menos de 0.9. En síntesis, se puede decir que el modelo de medida tiene un ajuste aceptable. Por lo tanto, se confirma la validez convergente.

Tabla 1

Análisis factorial confirmatorio del modelo.

\begin{tabular}{|c|c|c|c|c|}
\hline Variable & $\lambda$ & $\mathbf{t}$ & $\lambda$ estándar & $\begin{array}{c}\text { Índices de nivel } \\
\text { de ajuste }\end{array}$ \\
\hline V1F1 & $2.235^{\star * *}$ & 6.364 & 0.611 & \\
\hline V2F1 & $2.001^{* * *}$ & 6.253 & 0.598 & $X^{2}(15)=20.723$ \\
\hline V3F1 & $2.892^{* * *}$ & 9.685 & 0.832 & \\
\hline V4F1 & $2.869^{* * *}$ & 10.313 & 0.765 & BBNFI=0.922 \\
\hline V5F1 & $1.478^{* * *}$ & 5.134 & 0.478 & BBNNFI=0.958 \\
\hline V6F1 & $1.924^{* * *}$ & 5.579 & 0.536 & $\mathrm{CFI}=0.972$ \\
\hline V7F2 & $3.214^{* * *}$ & 13.845 & 1.000 & $\mathrm{GFI}=0.949$ \\
\hline V8F3 & $3.001^{* * *}$ & 13.741 & 1.000 & $A G F I=0.887$ \\
\hline V9F3 & $2.147^{\star * *}$ & 7.641 & 0.687 & SRMR $=0.052$ \\
\hline $\mathrm{V} 10 \mathrm{~F} 3$ & $2.168^{\star * *}$ & 7.649 & 0.695 & \\
\hline \multicolumn{4}{|c|}{$\begin{array}{l}\text { Niveles de significación: }{ }^{*} p<0.5 ;{ }^{* *} \mathrm{p}<0.1 ;{ }^{* \star *} \mathrm{p}<0.001 ; \text { (basado en } \mathrm{t}_{(499)} \text { dos colas) } \\
\mathrm{t}_{(0.05,499)}=1.964 \\
\mathrm{t}_{(0.01,499)}=2.585 \\
\mathrm{t}_{(0.001,499)}=3.291\end{array}$} & $\begin{array}{l}\text { F1=aprendizaje } \\
\text { de conocimientos } \\
\text { F2=codificación } \\
\text { F3=complejidad }\end{array}$ \\
\hline
\end{tabular}


El conocimiento como recurso estratégico para el aprendizaje interorganizativo Benavides Espinosa, María del Mar

Analizando la validez discriminante comparando las correlaciones de los factores y el coeficiente Alpha de Cronbach de cada uno de ellos (Sánchez y Sarabia, 1999) se comprueba que también se cumple.

Una vez determinadas las propiedades psicométricas de la escala de medida, se pasa a analizar ya las relaciones propuestas en las hipótesis que se han elaborado en el marco teórico. Para este análisis, se propone un modelo estructural en donde se recoge las relaciones causales, utilizando modelos de ecuaciones estructurales (Structural Equations Models, SEM) (Figura 1).

En concreto, para la primera de las hipótesis, la $\mathrm{H} 1$, se establece una regresión, donde F1 (aprendizaje de conocimientos) es la variable dependiente y F2 (codificación del conocimiento) la variable independiente; y para la segunda de las hipótesis, la H2, F1 es la variable dependiente y la F3 (complejidad del conocimiento) es la variable independiente de forma simultánea. Se calculan los estadísticos correspondientes a estas relaciones causales con el programa EQS 6.1 y se analiza el modelo estructural estable- cido según los resultados que se reflejan en la Tabla 2.

Los indicadores de la bondad de ajuste del modelo estructural, son más que aceptables, al igual que los estadísticos, para contrastar las relaciones planteadas en esta investigación.

No se puede confirmar la $\mathrm{H} 1$, pues el estadístico $t$ no es significativo, como se puede ver en la Tabla 2. Este hecho, hace pensar que la dificultad de codificación, en el caso de la joint venture, se ve compensada por la voluntad del socio que posee el conocimiento en enseñar al socio que aprende (normalmente, los conocimientos aprendidos serán los conocimientos pactados en el acuerdo). Este hecho es debido a que el socio de la joint venture que posee el conocimiento estratégico con un alto componente de "tacidad", puede facilitar su transferencia e incluso su asimilación, ya que los recursos comprometidos en una cooperación de este tipo son bastante elevados, minimizando así el efecto de la "tacidad". Cuando el compromiso entre los socios es menor, como ocurre en otras formas de cooperación, la importancia de la no codificación del conocimiento es mayor.

\section{Figura 1}

Modelo estructural propuesto.

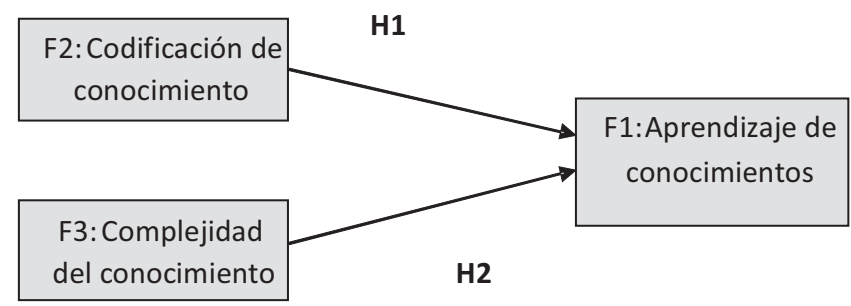

Fuente: Elaboración propia. 
Tabla 2

Parámetros estimados para las variables.

\begin{tabular}{llcc}
\hline \multicolumn{1}{c}{ Hipótesis } & Influencia & $\begin{array}{c}\text { Cargas } \\
\text { estandarizadas }\end{array}$ & t \\
\hline $\begin{array}{l}\text { H1: El establecimiento de una joint } \\
\text { venture facilita la transferencia de } \\
\text { conocimiento tácito generando así un } \\
\text { mayor aprendizaje de conocimientos }\end{array}$ & $\begin{array}{l}\text { Codificación en } \\
\text { aprendizaje de } \\
\text { conocimientos }\end{array}$ & 0.114 & 0.954 \\
estratégicos. & & & \\
H2: El establecimiento de una joint & Complejidad en & $0.235^{* *}$ & 2.767 \\
venture facilita la transferencia de & aprendizaje de & & \\
conocimiento complejo generando asi & conocimientos & & \\
un mayor aprendizaje de conocimientos & & & \\
estratégicos. & & & \\
$X^{2}(16)=21.857 ;$ & & \\
GFI=0.935, & & \\
AGFI=0.947, & & \\
SRMR=0.061 & & \\
\hline
\end{tabular}

Fuente: Elaboración propia.

Sin embargo, se puede confirmar la $\mathrm{H} 2$, y además, decir que es bastante significativa, pues t es mayor que 1.96. Analizando la complejidad del conocimiento, se ve que de los dos aspectos que se habían previsto para ella, han sido validados, tanto su aspecto técnico, como su aspecto social.

Estos resultados son coherentes con los de Cohen y Levinthal (1990) quienes afirman que las características que afectan a la facilidad de aprendizaje son básicamente dos: la complejidad del conocimiento asimilado y el grado en el que el conocimiento externo es adecuado a las necesidades de la empresa. Así pues, será de vital importancia aquellos conocimientos estratégicos, especialmente los complejos, que se puedan adquirir, ya que éstos pueden conducir a la generación de ideas que conduzcan a innovaciones radicales (Castiaux, 2007), y por lo tanto, crear o mantener la ventaja compe- titiva del socio que aprende estos conocimientos.

Esto conduce a pensar que cuando una empresa ha decidido qué conocimiento necesita para mantener o incrementar su ventaja competitiva, la cuestión es saber sus características, si ese conocimiento es muy complejo, y además, saber su grado de codificación. Si esto es así y no está disponible en el mercado, una empresa no podrá adquirirlo por sí sola, necesitará la ayuda de la organización que lo posea.

La joint venture puede ser una herramienta válida para aprender conocimientos complejos que estén pocos codificados, ya que el compromiso de los socios implicados en este tipo de cooperaciones ayudan a superar la barrera de la complejidad en mayor o menor medida dependiendo de lo codificado que se encuentre el conocimiento objeto de aprendizaje. 


\section{Conclusiones}

De la parte teórica de este trabajo, cabe deducir que la especificidad del conocimiento no es en sí misma una dimensión sino que depende del propio conocimiento. Por lo tanto, en este estudio, por un lado, se considera como principales dimensiones del conocimiento para su transferencia: la codificación y la complejidad. Y por otro lado, para ser considerados conocimientos estratégicos, según la Teoría de Recursos y Capacidades, deben ser poco o nada movibles y además difíciles de imitar. Además, se observa que hay relación entre ellas, tal que la codificación está relacionada con la movilidad, y la complejidad con la posibilidad de imitación.

Entonces, el problema es cómo aprender conocimientos estratégicos cuando la empresa no los posee y no están disponibles en el mercado. Por lo que una solución, es la utilización de acuerdos de cooperación. Ahora bien, no todas las formas de cooperación son iguales de convenientes para el aprendizaje, y más para la adquisición de conocimientos complejos y tácitos. En este trabajo, se va más allá, y se demuestra como una joint venture puede facilitar el aprendizaje de conocimiento estratégico, más o menos codificado, ya que posibilita principalmente la asimilación de conocimiento complejo, pues tiene el compromiso y la ayuda del socio que lo posee.

La complejidad del conocimiento, en sus dos aspectos (técnico y social), influye de manera positiva en el aprendizaje de conocimientos, pues a mayor complejidad más cantidad de conocimientos aprendidos, independientemente de que el proce- so de aprendizaje sea más costoso. Pues la relación que tiene el conocimiento con otros conocimientos (complejidad técnica) y la dependencia de ese conocimiento respecto a las personas o sistemas en donde esté localizado (complejidad social) determina la cantidad de conocimientos aprendidos y la cantidad de conocimientos nuevos que se pueden generar de éstos, incrementando así la capacidad de innovación.

La estrecha colaboración de un socio con otro en una joint venture, hace que un socio enseñe al otro ese conocimiento complejo ayudándole en su asimilación para su posterior aplicación. Por lo tanto, un socio puede aprender más conocimiento estratégico, y de forma más rápida con una joint venture que con otro tipo de estrategia. En este caso, se demostro que este tipo de cooperación es una forma efectiva para facilitar la transferencia de conocimiento complejo.

Respecto a la codificación, el obstáculo que pueda originar el grado de "tacidad" de un conocimiento estratégico en su transferencia a través de la joint venture, puede ser solventado, mediante la observación directa y un mayor contacto con el socio.

En relación a las implicaciones para los directivos, resalta la importancia de la adquisición de conocimientos a través de una joint venture, ya que permiten la obtención de conocimientos que pueden ser básicos para el desarrollo de competencias críticas generando así ventajas competitivas. Por ejemplo, una joint venture es un instrumento adecuado para la internacionalización de la empresa cuando ésta crea una joint venture con un socio local para aprender cómo funcio- 
na el nuevo mercado (adquisición de "conocimiento del mercado local" el cual es complejo y tácito) en el que se quiere introducir. La entrada en un nuevo mercado puede ser vital para mantener su ventaja competitiva y la joint venture le facilita esta opción estratégica.

En un futuro, se quiere profundizar en el estudio de las dimensiones del conocimiento y en la implicación de estas con otros tipos de cooperaciones, e incluso con otras formas de estrategia que conlleve el aprendizaje de conocimientos estratégicos.

\section{Referencias bibliográficas}

Abarca Oriester, Francisco (2010). Alianzas estratégicas: ¿Adquisición o acceso al conocimiento?. Ciencias Económicas, Vol. 28, No. 2, pp. 263-287.

Amit, Raphael y Schoemaker, Paul J.S. (1993). Strategic Assets and Organizational Rent. Strategic Management Journal, vol. 14, No. 1, pp. 33-46.

Badaracco, Joseph L. (1992). Alianzas estratégicas: El caso de General Motors e IBM. Mc Graw-Hill. Madrid.

Benavides, María del Mar (2007). El impacto de la joint venture en el aprendizaje interorganizativo. Tesis doctoral de la Universitat de Valencia.

Bianchi, Mattia; Chiesa, Vittorio y Frattini, Federico (2011). Selling technological knowledge: Managing the complexities of technology transactions. Reseach Technology Management, Vol. 54, No. 2, pp. 18-26.

Blasco, José Luis y Grimaltos, Tobias (1997). Teoria del coneixement. Servei de Publicacions. Universitat de València.

Burns, Alan T.; Acar, William y Datta, Pratin (2011). A qualitative exploration of entrepreneurial knowledge transfers.
Journal of Knowledge Management, Vol 15, No. 2, pp. 270-298.

Castiaux, Annick (2007). Radical innovation in Established Organizations: Being a Knowledge Predator. Journal of Engineering and technology Management, Vol. 24, No.1-2, pp. 36-52.

Cohen, Wesley M. y Levinthal, Daniel A. (1990). Absorptive Capacity: A New Perspective on Learning and Innovation. Administrative Science Quarterly, Vol. 35, pp. 128-152.

Dierickx, Ingemar y Cool, Karel (1989). Asset Stock Accumulation and Sustainability of Competitive Advantage. Management Science, Vol. 35, pp. 15041511.

Grant, Robert M. (1996). Toward a Knowledge-Based Theory of the Firm. Strategic Management Journal, Vol. 17, winter special issue, pp. 109122

Hair, Joseph F.; Anderson, Rolph E.; Tatham, Ronald L. y Black, William C. (1999). Análisis Multivariante. Prentice Hall, Madrid.

Hedlund, Gunnart (1994). A Model of Knowledge Management and the $\mathrm{N}$-form Corporation. Strategic Management Journal, Vol. 15, pp. 73-90.

Hennart, Jean-Francois. (1988). A Transaction Costs Theory of Equity Joint Ventures. Strategic Management Journal, Vol. 9, pp. 361-374.

Huang, Qian; Davison, Robert y Gu, Jibao (2008). Impact of personal and cultural factors on knowledge sharing in China. Asia Pacific Journal Management, Vol. 25, No. 3, pp. 451-471.

Inkpen, Andrew C. (1998). Learning and Knowledge Acquisition through International Strategic Alliances. The Academy of Management Executive, Vol. 12, No. 4, pp. 69-80.

Inkpen, Andrew C. (2000). Learning through Joint Ventures: A Framework of 
El conocimiento como recurso estratégico para el aprendizaje interorganizativo Benavides Espinosa, María del Mar

Knowledge Acquisition. Journal of Management Studies, Vol. 37, No. 7, pp. 1019-1043.

Inkpen, Andrew C. (2008). Knowledge transfer and international joint ventures: the case of NUMMI and Generals Motors. Strategic Management Journal, Vol. 29, No. 4, pp. 447-453.

Inkpen, Andrew C. y Beamish, Paul W. (1997). Knowledge Bargaining Power, and the Instability of International Joint Ventures. Academy of Management Review, Vol. 22, pp. 177-202.

Inkpen, Andrew C. y Dinur, Adva (1998). Knowledge Management Processes and International Joint Ventures. Organization Science, Vol. 9, No. 4, pp. 454-468.

Itami, Hiroyuki y Roehl, Thomas W. (1987). Mobilizing Invisible Assets. Havard University Press, Cambridge, MA.

Jensen, Michael y Meckling, William (1992). Specific and General Knowledge and Organizational Structure. En Werin, L. and Wijkander, H. (eds.): Contracts Economics. Oxford\&Cambridge, Blackwell, Massachusetts, pp. 251291.

Kogut, Bruce y Zander, Udo (1992). Knowledge of the Firm, Combinative Capabilities and the Replication of Technology. Organization Science, Vol. 3, No. 3, pp. 383-397.

Kogut, Bruce y Zander, Udo (1995). Knowledge and the Speed of the Transfer and Imitation of Organizational Capabilities: An Empirical Test. Organizational Science, Vol. 6, No. 1, pp. 76-92.

Lin, Hsiu-Fen (2007). Knowledge sharing and firm innovation capability: An empirical study. International Journal of Manpower, Vol. 28, No.3/4, pp. 315-332.

Lyles, Marjorie A. y Salk, Jane E. (2007). Knowledge adquisition from foreign parents in international joint ventures: an empirical examination in the Hun- garian context. Journal of International Business Studies, Vol. 38, No. 1, pp. 3-18.

Minbaeva, Dana (2007). Knowledge transfer in multinationals. Management International Review, Vol. 47, No. 4, pp. 567-593.

Mowery, David C.; Oxley, Joane E. y Silverman, Brian S. (1996). Strategic Alliances and Interfirm Knowledge Transfer. Strategic Management Journal, Vol. 17, winter special issue, pp. 77-91.

Nonaka, Ikujiro (1994). A Dynamic Theory of Organizational Knowledge Creation. Organization Science, Vol. 5, No. 1, pp. 14-37.

Nonaka, Ikujiro y Takeuchi, Hirotaka. (1995). The Knowlegde-Creating Company, How Japanese Companies Create the Dynamics of Innovation, Oxford University Press, New York.

Pak, Yong Suhk y Park, Young-Ryeol (2004), A Framework of Knowledge Transfer in Cross-Border Joint Ventures: An Empirical Test of the Korean Context. Management International Review, Vol. 44, No. 4, pp. 435-455.

Peteraf, Margaret A. (1993). The Cornerstones of Competitive Advantage: A Resource-Based View. Strategic Management Journal, vol. 14, No. 3, p. $179-191$.

Polanyi, Michael (1967). The Tacit Dimension. Anchor Books. Garden City, Doubleday, New York.

Reed, Richard y DeFillippi, Robert J. (1990). Causal Ambiguity, Barriers to Imitation, and Sustainable Competitive Advantage. Academy of Management Review, Vol. 15, No. 1, pp. 88-102.

Rumelt, Richard P. (1984). Towards a Strategic Theory of the Firm. En Foss, Nicolai J. (ed., 1997). Resources, Firms and Strategies. A Reader in the Resource-Based Perspective. Ox- 
ford University Press, New York, p. 131-145.

Rumelt, Richard P. (1987). Theory, Strategy and Entrepreneurship. En Teece, David J. (ed.): The Competitive Challenge: Strategic for Industrial Innovation and Renewal. Ballinger Publishing Company, Cambridge, MA, pp. 137-158.

Sánchez, M. y Sarabia, Francisco José (1999). Validez y fiabilidad de escalas. En Sarabia, Francisco José (ed.): Metodología para la investigación en Marketing y Dirección de Empresas. Editorial Pirámide S.A. Madrid, pp. 363-393.

Simonin, Bernard L. (1999). Ambiguity and the Process of Knowledge Transfer in Strategic Alliances. Strategic Management Journal. Vol. 20, No. 7, pp. 595-623.

Spender, J.C. (1994). Organizational Knowledge, Collective Practice and Penrose Rents. International Business, Vol. 3, No. 4, pp. 353-367.

Subramaniam, Mohan y Venkatraman, Venkat N. (2001). Determinants of transnational new product development capability: testing the influence of transferring and deploying tacit overseas knowledge. Strategic Management Journal, Vol. 22, No. 4, pp. 359-378.
Swift, Peter y Hwang, Alvin (2008). Learning, dynamic capabilities and operating routines: A consumer package goods company. The Learning Organization, Vol. 15, No. 1, pp. 75-95.

Wahab, Sazali Adbul; Abdullah, Haslinda; Uli, Jegak; Rose, Raduan Che (2010). Inter-firm technology transfer and performance in international joint venture firms. International Journal of Business and Management. Vol. 5, No. 4, pp. 93-103.

Winter, Sidney G. (1987). Knowledge and Competence as Strategic Assets. En Teece, D.J. (ed.,): The Competitive Challenge: Strategic for Industrial Innovation and Renewal. Ballinger Publishing Company. Cambridge, MA, pp. 159-184.

Wonglimpiyarat, Jarunee (2005). Does complexity affect speed of innovation? Technovation, Vol. 25, pp. 865-882.

Wruch, Karen Hopper, Jense, Michael C. (1994). Science Specific Knowledge and Total Quality Management. Journal of Accounting and Economics, Vol. 18, pp. 247-287.

Zander, Udo y Kogut, Bruce (1995). Knowledge and the Speed of the Transfer and Imitation of Organizational Capabilities: An Empirical Test. Organization Science, Vol. 6, No. 1, pp. 76-92. 